\title{
Underwater Vehicle CFD Analyses and Reusable Energy Inspired by Biomimetic Approach
}

\author{
Nedelcu Andra-Teodora 1 ${ }^{*}$, Faităr Cătălinn ${ }^{2}$,Liviu-Constantin Stan ${ }^{3}$ and Nicolae Buzbuchi ${ }^{4}$ \\ ${ }^{1}$ Mircea cel Batran Naval Academy, Faculty of Navigation and Naval Transport, Fulgerului Street, 1, \\ RO-900218, Constanta, Romania ; andra.nedelcu@anmb.ro \\ 2,3,4 Maritime University of Constanta, Faculty of Naval Electromechanics, 104 Mircea cel Batran \\ Street, 900663, Constanta, Romania; catalinfaitar@yahoo.ro; liviu_stan14@yahoo.com; \\ nicolae_buzbuchi@yahoo.com \\ * Correspondence: andra.nedelcu@anmb.ro
}

\begin{abstract}
Over the last few decades, ocean research and exploration have made underwater mechanical systems a necessity. Underwater vehicles provide a new kind of marine platforms that could represent a great necessity in many areas of oceanographic research. Until now, the underwater vehicles come in a verity of shapes, sizes and means of propulsion. Depending on these characteristics, the type and mission of the vehicle are also determined.

The underwater robots are used for different inspection and intervention missions in e.g. the oil and gas industry, ocean science research. Due to multiple applications to which the vehicle can participate, it can be successfully used and to determine methods of re-use of marine energy. Environmental mapping provides accurate information about the main areas of interest of the energy, as well as the exploitation possibilities of that.

Most of the time, biomimetic robots were inspired their senso structure, from different kind of animals, such as insects, fish and birds. Nowadays, the concept of a underwater robotic vehicles capable to move independently, autonomously or remotely, has a great potential and a large application. This is the reason that the last studies have been directed on biomimetic robots. The fish and other underwater animals have evolved superior swimming capabilities in many ways and represent a starting point to explain the fluid-mechanical principles. Furthermore, the underwater animals develop and achieve extraordinary propulsion efficiencies, acceleration and maneuverability. They can also achieve high speed under water. Implanting and creating a vehicle through a biomimetic approach reduces the energy used to maneuver the vehicle as it can automatically correct its position and displacement. The paper presents an examination of the state of biomimetic robotic fishes, underlining the reason why bio-inspiration can help us in the underwater locomotion technology.
\end{abstract}

Keywords: biomimetic, underwater, CFD method.

\section{Introduction}

The underwater vehicle have a large range of applications in marine geosciences. Their interest are increasingly also in scientific, military, commercial and policy sectors. They are well suited to 
exploration of extreme environments, from the world`s deepest hydrothermal vents to beneath polar ice sheets.

Over the last few decades, ocean research and exploration have made underwater mechanical systems a necessity. Underwater vehicles provide a new kind of marine platforms that could represent a great necessity in many areas of oceanographic research. Until now, the underwater vehicles come in a verity of shapes, sizes and means of propulsion. Depending on these characteristics, the type and mission of the vehicle are also determined.

The primary applications in marine geosciences that have resulted from underwater vehicle are the studies of submarine volcanism and hydrothermal vent, mapping and monitoring of low-temperature fluid escape features and chemosynthetic ecosystems and benthic habitat mapping in shallow and deep-water environments.

The underwater vehicle have also a particular importance in scientific field.

Studying the energy of the waves and the impact it has on the body of the vehicle, we can automatically correct the movement and movement of the body into the water.

Most of the time, biomimetic robots were inspired their senso structure, from different kind of animals, such as insects, fish and birds. Nowadays, the concept of a underwater robotic vehicles capable to move independently, autonomously or remotely, has a great potential and a large application. This is the reason that the last studies have been directed on biomimetic robots. The fish and other underwater animals have evolved superior swimming capabilities in many ways and represent a starting point to explain the fluid-mechanical principles. Furthermore, the underwater animals develop and achieve extraordinary propulsion efficiencies, acceleration and maneuverability. They can also achieve high speed under water. The paper presents an examination of the state of biomimetic robotic fishes, underlining the reason why bio-inspiration can help us in the underwater locomotion technology.[2]

Starting with the ROV body vehicle, this paper presents the possibility to improve his locomotion and propulsion inspired by biomimetic approach.

Most of the robotic fish develop bionic wave propulsion, but the ROV did not perform as well as fish in terms of swimming capability. The improvement of the performance still an ongoing work.

The main reason in this paper is to show the ability of pressure sensing on imitating the function of lateral line systems. In the literature of specialty, the pressure sensors include dipole source localization, object recognition and flow pattern detection. Attaching an artificial lateral line system over vehicle body should be beneficial for the performance improvement of underwater vehicle. This lateral line helps vehicles to optimize energy and improve the performance of motion control. The pressure distribution over body vehicle are used to estimate the orientation of streamline body. For example, Salumae and Kruusmaa apply pressure sensing to achieve the flow-relative and flow-aided navigation. ${ }^{[1]}$

In Figure 1 are presented the lateral line on a different type of fishes. Many fish species have one or more lateral line. 

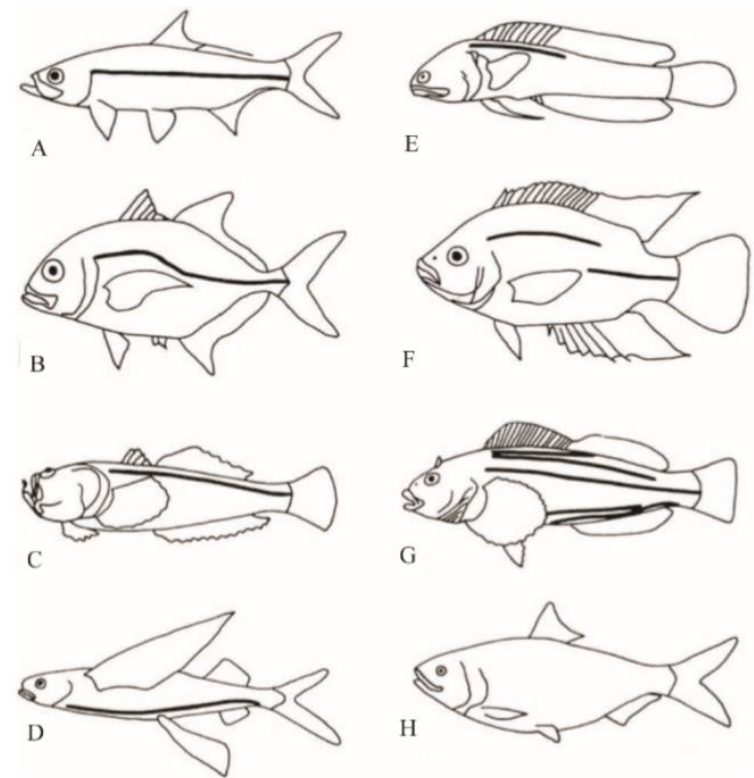

Figure 1. Lateral line along fish (A)Complete straight; (B) Complete arched; (C) Dorsally placed; (D) Ventrally placed; (E) Incomplete; (F) Disjunct; (G) Multiple and(H) Absent.

This paper presents a Computational Fluid Dynamic method for solving and analysis problems involving a fluid flow by numerical simulations. The simulation generates numerical data used to describe the hydrodynamics characteristics of vehicle.

Firstly, is solved the numerical hydrodynamics characteristics to provide pressure distribution over the body vehicle. Secondly, we are searching for more virtual pressure sensors inspired by lateral line of fish that could be used to collect information about pressure distribution over vehicle body. Finally, we validate the experiment by attaching the sensors over body vehicle and used the CFD simulation and also compare the results.

\section{CFD method}

The fish lateral line is used to sense flow information and avoid the underwater obstacle. CFD method is a computational hydrodynamics way solve Navier-Stokes equation by means of computer-based information using Ansys software. Computers are used to prepare the data, build computational domain and mesh, perform numerical solution of the equations, and to analyze the solution results. The simulation process involves three main stages:

- Pre-processing

- Solving

- Post-processing

The first stage, pre-processing, enables the input data and setup of the flow simulation problem. In this study, the geometry are modeled using SolidWorks program. Figure 2 presents the geometry of the vehicle.

The 3D modeling of mechanical systems for submarine vehicles implies the accurate geometric design of the components, the restrictions and the operating conditions.

The 3D geometric model classification is as follows:

- wireframe; 
- surface oriented (surface model);

- volume-oriented (solid model).

3D models are obtained by the following techniques:

- solid construction geometry (CSG);

- through borders (B-rep);

- by spatial enumeration (octree ...);

- sweeping, etc.

- analytical (ASM);

- parametric / procedural.

The shape of the 3D geometric models is based on a set of elementary shapes - prism, cylinder, cone, sphere - from the combination of which (the boolean operations) result the desired pattern.

These forms are called primitive and are found in any CAD application that allows 3D modeling. Primitives are parameterized, that means their dimensions can be changed.

3D geometric modeling uses a series of terms such as:

- creating - the workpiece geometry generation operation in the modeling space;

- construction - the operation by which an entity is used to modify another, the result being a new entity;

- modification - the operation of changing the dimensional values of an entity.

The geometric model of a piece is derived from a primitive to which its constructive and technological characteristics change. Changes are made by applying a series of special operators: offset, thicken, loft, extrude, sweep.

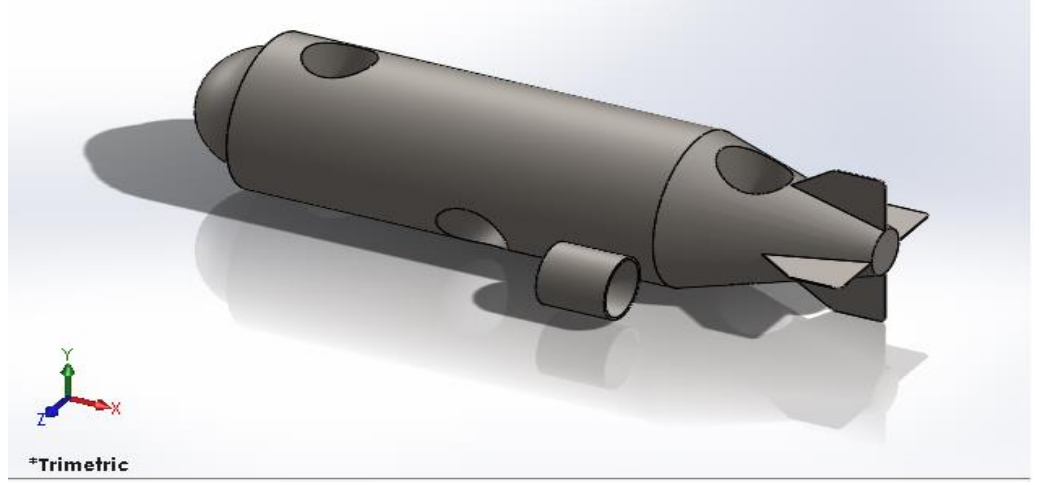

Figure 2. SolidWorks generate

Before performing the CFD, the mesh size needs to be defined properly as the shape of the boundary. A surface mesh is a discrete representation of the geometry of vehicle that will be used for the volume mesh generation. It is built of triangular faces. In this case, the surface mesh contains no free edges or mismatches, this means it is closed. Using ANSYS CFX software, in this case, the number of elements represented approximately 756330 elements. 


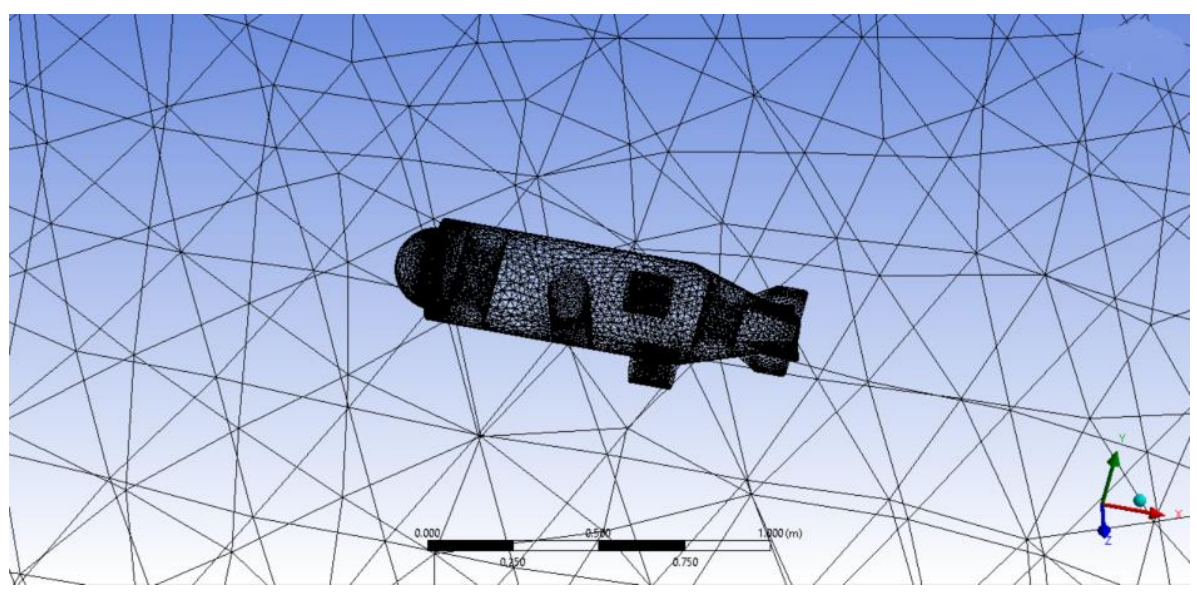

Figure 3. 3D surface vehicle in Solidworks program imported to a CFD mesh generation program

The properties of the fluid used for simulation are presented in Table 1.

Tabel 1. Fluid domain data

\begin{tabular}{ccc}
\hline Fluid domain data & Value & Units \\
\hline Density & 997.0 & {$\left[\mathrm{~kg} / \mathrm{m}^{3}\right]$} \\
Molar Mass & 18.02 & {$[\mathrm{~kg} / \mathrm{kmol}]$} \\
Specific Heat Capacity & 4181.7 & {$[\mathrm{~J} / \mathrm{kgK}]$} \\
Reference Pressure & 1 & {$[\mathrm{~atm}]$} \\
Reference Temperature & 25 & {$[\mathrm{C}]$} \\
Dynamic Viscosity & $8.899 \mathrm{E}-4$ & {$[\mathrm{~kg} / \mathrm{ms}]$} \\
Thermal Conductivity & 0.6069 & {$[\mathrm{~W} / \mathrm{mK}]$} \\
Absorption Coefficient & 1.0 & {$[1 / \mathrm{m}]$} \\
Thermal Expansivity & $2.57 \mathrm{E}-04$ & {$[1 / \mathrm{K}]$} \\
\hline
\end{tabular}

Figure 4 presents the boundary conditions. We chose a " no-slip" condition because water can not penetrate through the solid surface. The flow regime inside the sphere is subsonic, the vehicle reached a velocity of $2 \mathrm{~m} / \mathrm{s}$. 


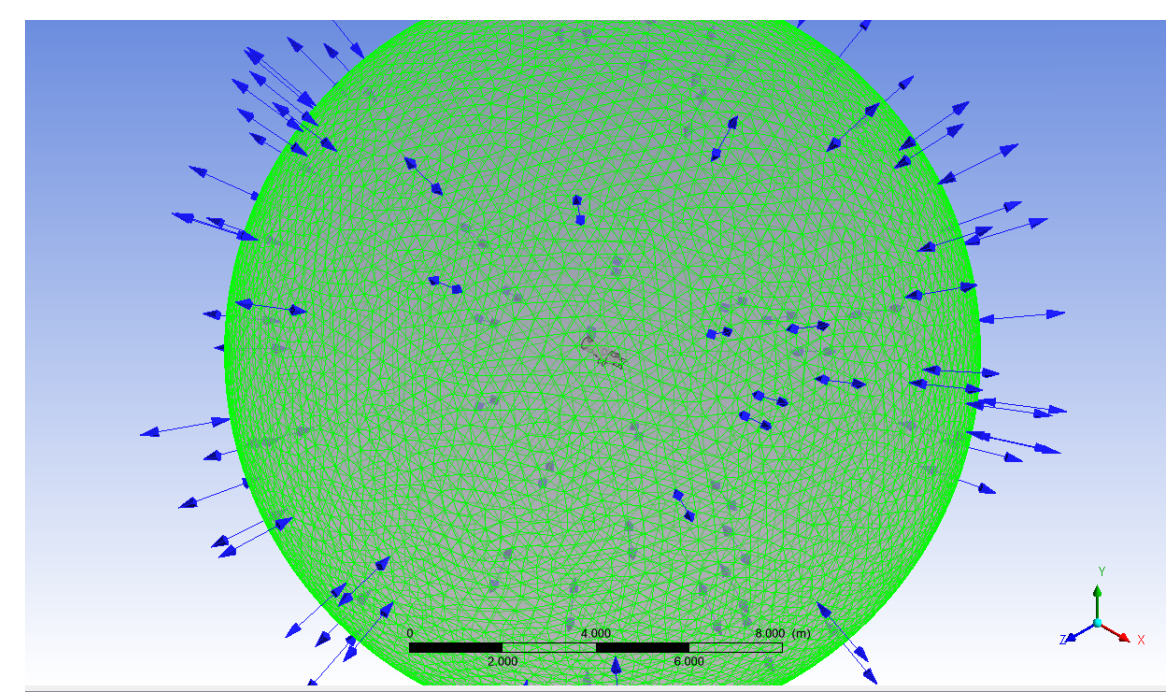

Figure 4. Boundary condition for domain

\section{Theoretical issues}

In igure 5 are presented the space-fixed coordinate system $\mathrm{x} 0 \mathrm{y} 0$ and the segment-fixed coordinate systems xiyi, $\mathrm{i}=1,2,3$. The figure presents a conceptual view of the body-spline shape, where $\theta_{0}$ represents the vehicle angle in frame $x_{0} y_{0} ; \theta_{1}$ represents the body vehicle angle in frame $x_{1} y_{1}$ and $\theta_{2}$ represenst the vehicle tail fin angle in frame $x_{2} y_{2}$. The coordinates could be transferred between systems take into consideration the relationships between the position and the angles. In "Fast continuous swimming of two pelagic predators" by Vieder and Hess in 1984 [4] , have revealed that the motion of the body-spline can be regarded as a traveling wave whose amplitude increases from the nose to the tail. Lighthill (1960) propose and presents a specific form of the traveling wave equation:

$$
y(x, t)=\left(c_{1} x+c_{2} x^{2}\right)(\sin (k x+\omega t)+o f f s e t)
$$



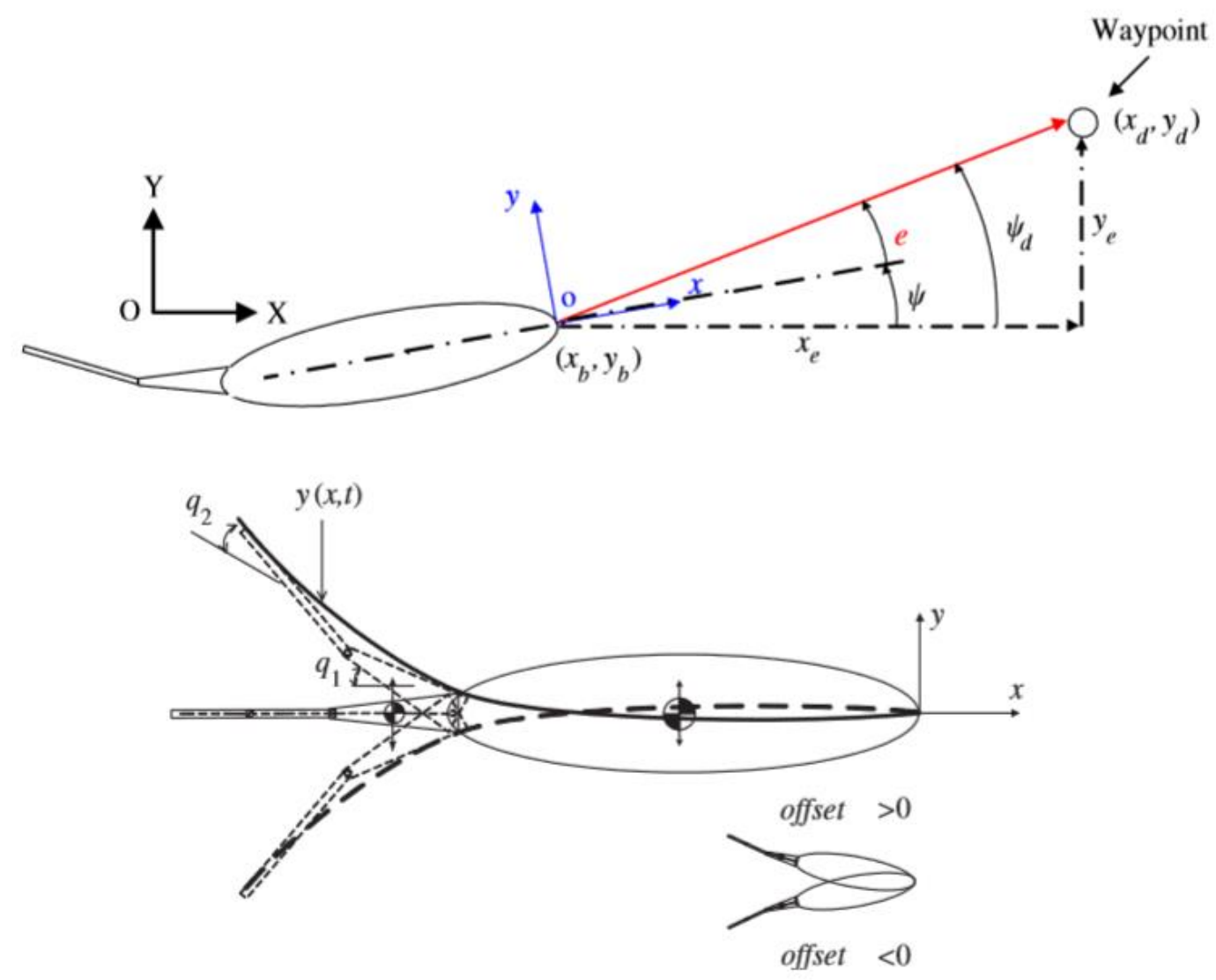

Figure 5. Definitions of coordinate systems

Where $y(x, t)$ represents the transverse displacement of the body, $x$ the displacement along the axis, $k=2 \pi / \lambda$ the body wave number, $\lambda$ is the body wavelength, $c_{1}$ the coefficient of linear curvature, $c_{2}$ the adrenal amplitude coefficient, $\omega \omega=2 \pi f=2 \pi / T$ frequency of body waves and $T$ wave period. The equation present a sinusoidal wave that action from the center of the $x_{1} y_{1}$ to the tail bounded by a second-order $c_{1} x+c_{2} x^{2}$ amplitude envelope. When the vehicle is turning, the parameter offset has a non-zero value.

It is also defined a fitness function as a basis for evaluating propulsion efficiency and determining an efficient swimming mode.

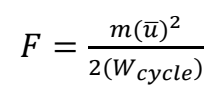

where $F$ represent a fitness function. A fitness function represent a particular type of objective function used to summarize, as a single figure of merit, how close a given design solution is to achieving the set aims. In this case, the fitness function represents the transmission efficiency of the drive system. It is defined as the ratio of the output swimming power to the power input to the propulsors. In equation (2), $m$ represent the vehicle; $\bar{u}$ is the averaged forward speed, and $W_{\text {cycle }}$ is the amount of work that is performed by the drive system in each undulation cycle. To determinate the forward speed of the vehicle we directly measurable from Doppler sonar signals.

For an underwater vehicle moving in deep water at a constant speed, without taking into consideration the surface water effect, the hydrodynamic forces and moments are caused by the fluid viscosity, which depend on the geometry and motion velocities. In general, they may be expressed as follows: 


$$
\begin{gathered}
\vec{F}_{D}=\vec{F}_{D}(\vec{U}) \\
\vec{F}_{D}=\{X, Y, Z, K, Z, M, N\} ; \vec{U}=\{u, v, w, p, q, r\}
\end{gathered}
$$

In this case, the component of the forces and moments can be expressed by the multivariate Taylor series of the velocities. For example, if we simplify the series to the second order, the forces and moments may be expressed as follows:

$$
\begin{array}{r}
X=X_{u} u+X_{u u} u^{2}+X_{w} w+X_{w w} w^{2} \\
Z=Z_{u} u+Z_{u u} u^{2}+Z_{w} w+Z_{w w} w^{2} \\
M=M_{u} u+M_{u u} u^{2}+M_{w} w+M_{w w} w^{2}
\end{array}
$$

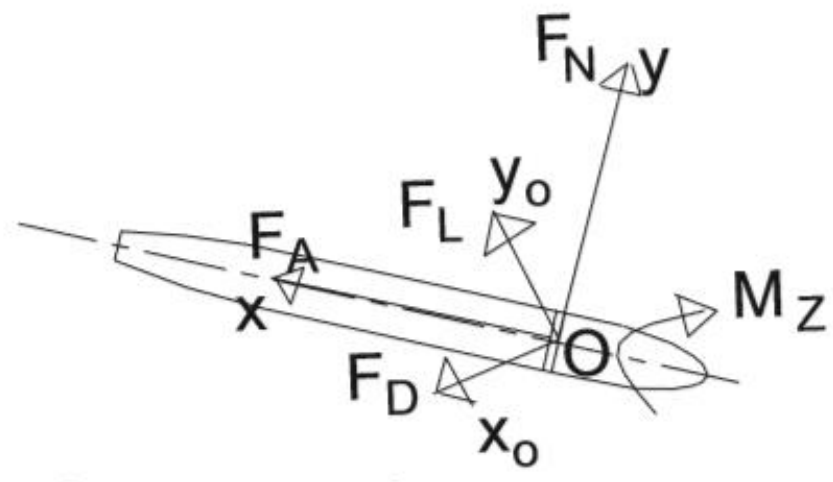

Figure 6. Body and coordinate systems and component forces

The final forces and moments acting on the model in the body coordinate system are obtained as follows:

$$
\begin{gathered}
\text { Axial force }\left(F_{A}\right)=A_{x} \\
\text { Normal force }\left(F_{N}\right)=N_{1}+N_{2}
\end{gathered}
$$

The axial and normal force coefficients are obtained as follows:

$$
\begin{aligned}
& C_{A}=F_{A} /\left(0.5 \rho U^{2} \nabla^{\frac{2}{3}}\right) \\
& C_{N}=F_{N} /\left(0.5 \rho U^{2} \nabla^{\frac{2}{3}}\right)
\end{aligned}
$$

To maintain uniformity in presentation of the force components both in experimental and numerical investigations, it is necessary to determinate the drag and lift forces. We resolve and optain the drag and lift forces on the body by resolving the axial and normal component of the forces from the body coordinate system presented in Figure 6.

$$
\begin{aligned}
& \text { Drag force }\left(F_{D}\right)=F_{A} \cos \alpha-F_{N} \sin a \\
& \text { Lift force }\left(F_{L}\right)=-\left(F_{N} \cos \alpha+F_{A} \sin a\right)
\end{aligned}
$$

The drag and lift moment coefficients are obtained as follows:

$$
\begin{aligned}
& C_{D V}=F_{D} /\left(0,5 \rho U^{2} \nabla^{2 / 3}\right) \\
& C_{L V}=F_{L} /\left(0,5 \rho U^{2} \nabla^{2 / 3}\right) \\
& C_{M V}=M_{Z} /\left(0,5 \rho U^{2} \nabla^{2 / 3} L\right)
\end{aligned}
$$

Where the $C_{D V}$ is drag force coefficient, $C_{L V}$ is lift force ccoefficient, $\rho$ is the density of water, $U$ is the towing speed, $\nabla$ is volume of the body, $L$ is length of the body and $\alpha$ is angle of attack in degrees. 


\section{Numerical simulations}

In order to achieve the underwater vehicle modeling approach, Figure 7 shows the overall approach from numerical modeling to control system design.

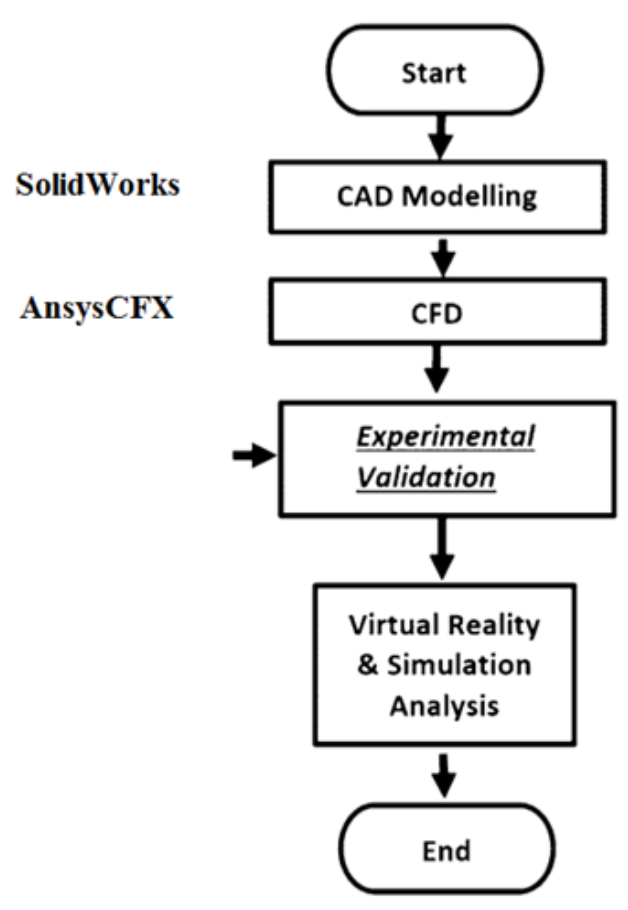

Figure 7. Chart of proposal systematic computation

The hydrodynamic forces on the body vehicle depends on the body construction. We considered the body vehicle used for CFD analysis for estimating the drag and lift forces for different speed combinations. The design particulars are presented in Table 1.

Table 1 Design particulars of the ROV

\begin{tabular}{ccc}
\hline Physical property & Value & Units \\
\hline Length overall & 1200 & {$[\mathrm{~mm}]$} \\
Diameter of body & 300 & {$[\mathrm{~mm}]$} \\
Diameter of propellers & 120 & {$[\mathrm{~mm}]$} \\
Number of propellers & 5 & {$[$ piece] } \\
Length of wings & 165 & {$[\mathrm{~mm}]$} \\
Number of wings & 4 & {$[$ piece] } \\
Diameter of tail & 90 & {$[\mathrm{~mm}]$} \\
\hline
\end{tabular}

The computational domain around the experimental hull body shown in Figure 9 and it is represented by a sphere with a radius of $7.5 \mathrm{~m}$, representing a volume of $1767,15 \mathrm{~m}^{3}$. 


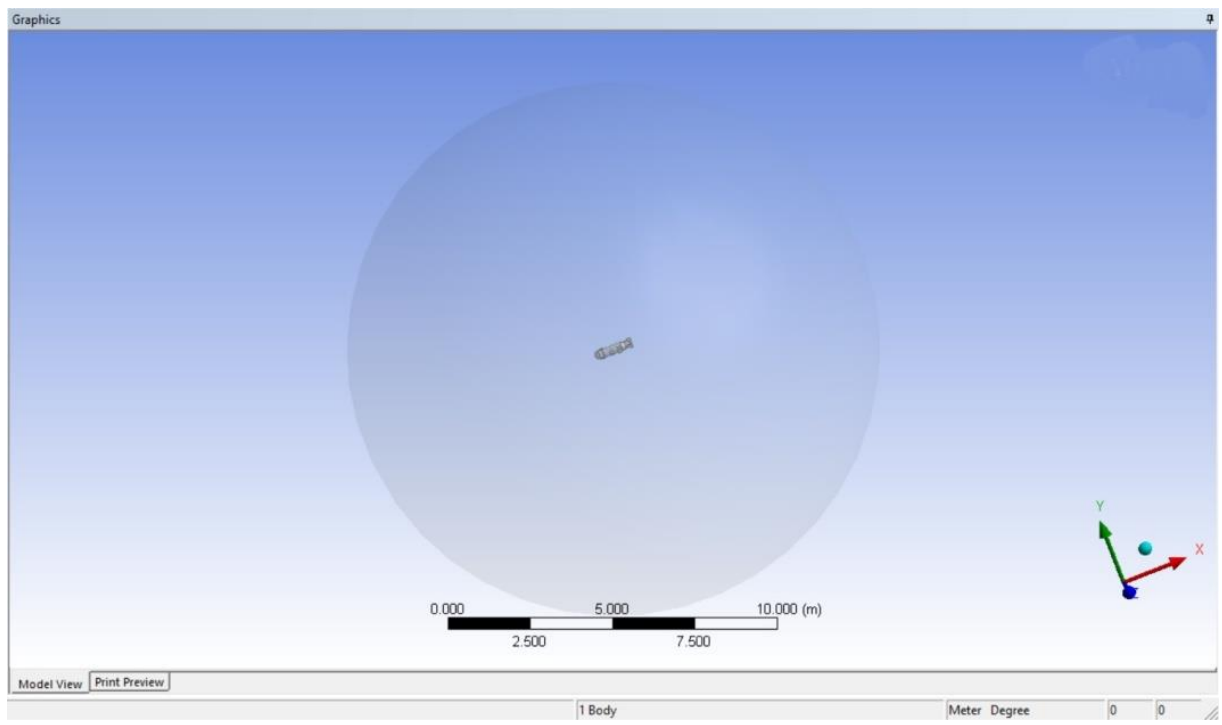

Figure 9. The domain around the underwater vehicle

During the simulation, the vehicle achieve a speed of $2 \mathrm{~m} / \mathrm{s}$. Other initial conditions are water temperature $25^{\circ} \mathrm{C}$ and a turbulent flow [5].

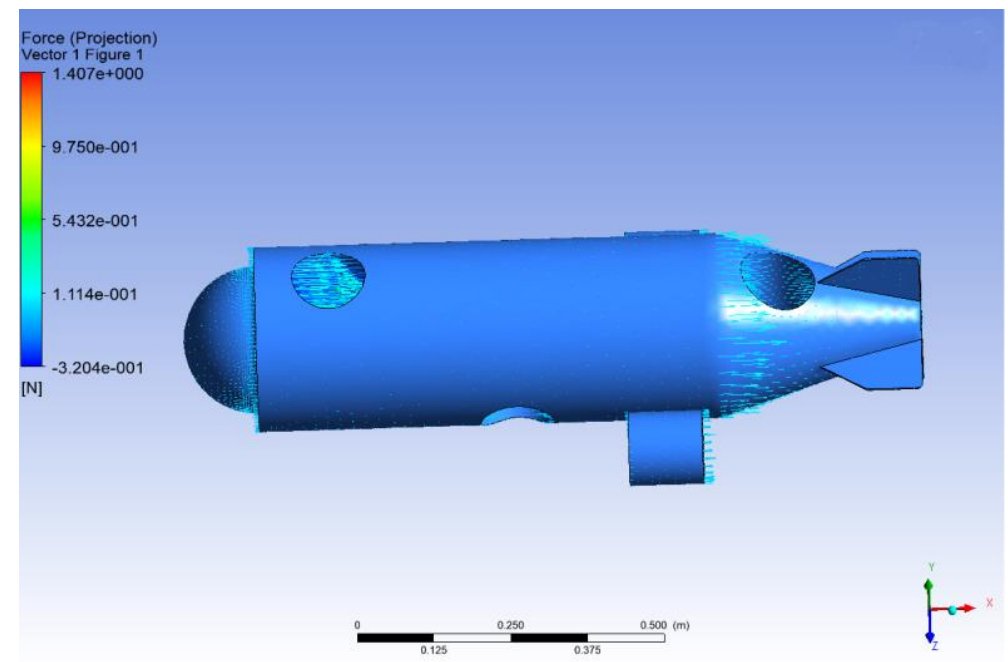

Figure 10. The force over the vehicle body

Figure 10 present the vector force concerning the vehicle body. After simulation, we observe an absolute pressure reaching a minimum value of 98107.5 [Pa], and maximum value of 103277 [Pa].

The total pressure value is between $-2726.72[\mathrm{~Pa}]$ along the body vehicle and $2347.92[\mathrm{~Pa}]$ around the holes for propulsions.

In the following table are presented the values for different variable for the domain (the sphere).

Table 2. Values for domain

\begin{tabular}{llll}
\hline \multicolumn{1}{c}{ Default Domain } & \multicolumn{1}{c}{ X-Comp. } & \multicolumn{1}{c}{ Y-Comp. } & \multicolumn{1}{c}{ Z-Comp. } \\
\hline Pressure Force On Walls & $7.5096 \mathrm{E}+01$ & $6.3069 \mathrm{E}-01$ & $-3.5662 \mathrm{E}+00$ \\
Viscous Force On Walls & $2.5405 \mathrm{E}+00$ & $6.8692 \mathrm{E}-02$ & $7.2804 \mathrm{E}-02$ \\
Pressure Moment On Walls & $1.7872 \mathrm{E}-01$ & $2.1962 \mathrm{E}+00$ & $1.4031 \mathrm{E}+00$ \\
Viscous Moment On Walls & $2.7021 \mathrm{E}-02$ & $-4.1126 \mathrm{E}-02$ & $1.0573 \mathrm{E}-01$ \\
\hline
\end{tabular}


Figure 11 present the velocity which reached a minimum value of $0.0167924[\mathrm{~m} / \mathrm{s}]$ concerned to the stern of vehicle, and a maximum value of $2.64293[\mathrm{~m} / \mathrm{s}]$

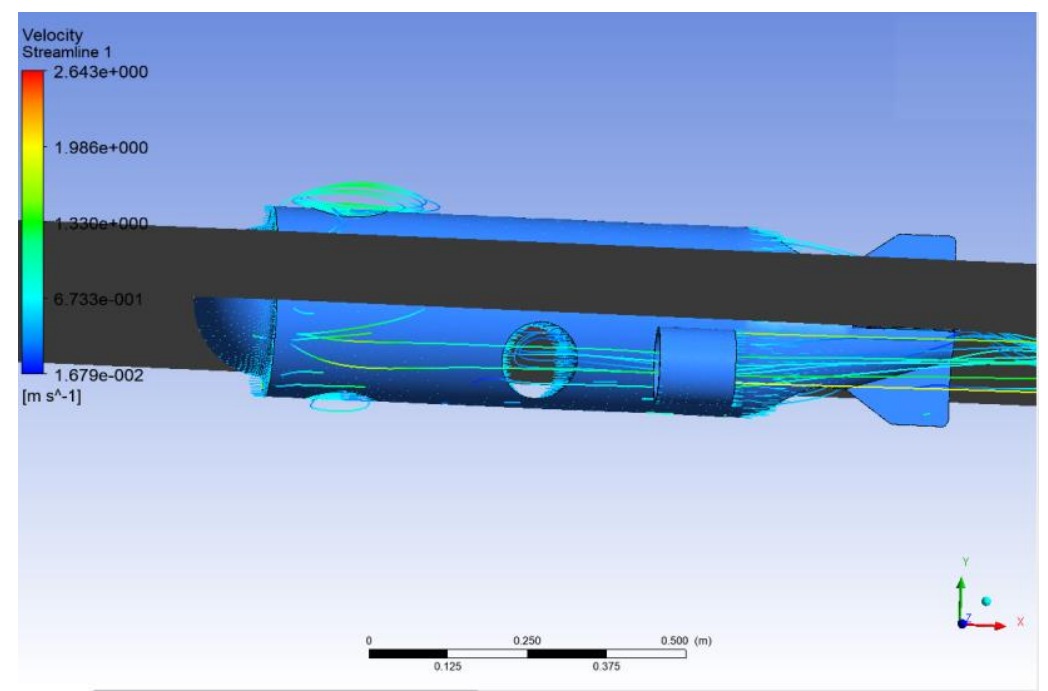

Figure 11. The velocity over vehicle body

In Figure 12 it is represented the graphic with the pressure distribution measured in equatorial plan of vehicle. We observed an irregular distribution of pressure in equatorial plan of vehicle. For the first $0.1 \mathrm{~m}$, the pressure suffer a decrease, after that the pressure increase reaching $1.500 \mathrm{~Pa}$ value. After the increase moment, the pressure decrease, reaching the most small value $-1.900 \mathrm{~Pa}$.

The simulation results that the pressure distribution keeps its longitudinal axis parallel to itself and so determine different curves on port and starboard side. The difference are not very bigin the order of Pascals- but still measurable with the appropriate sensors.

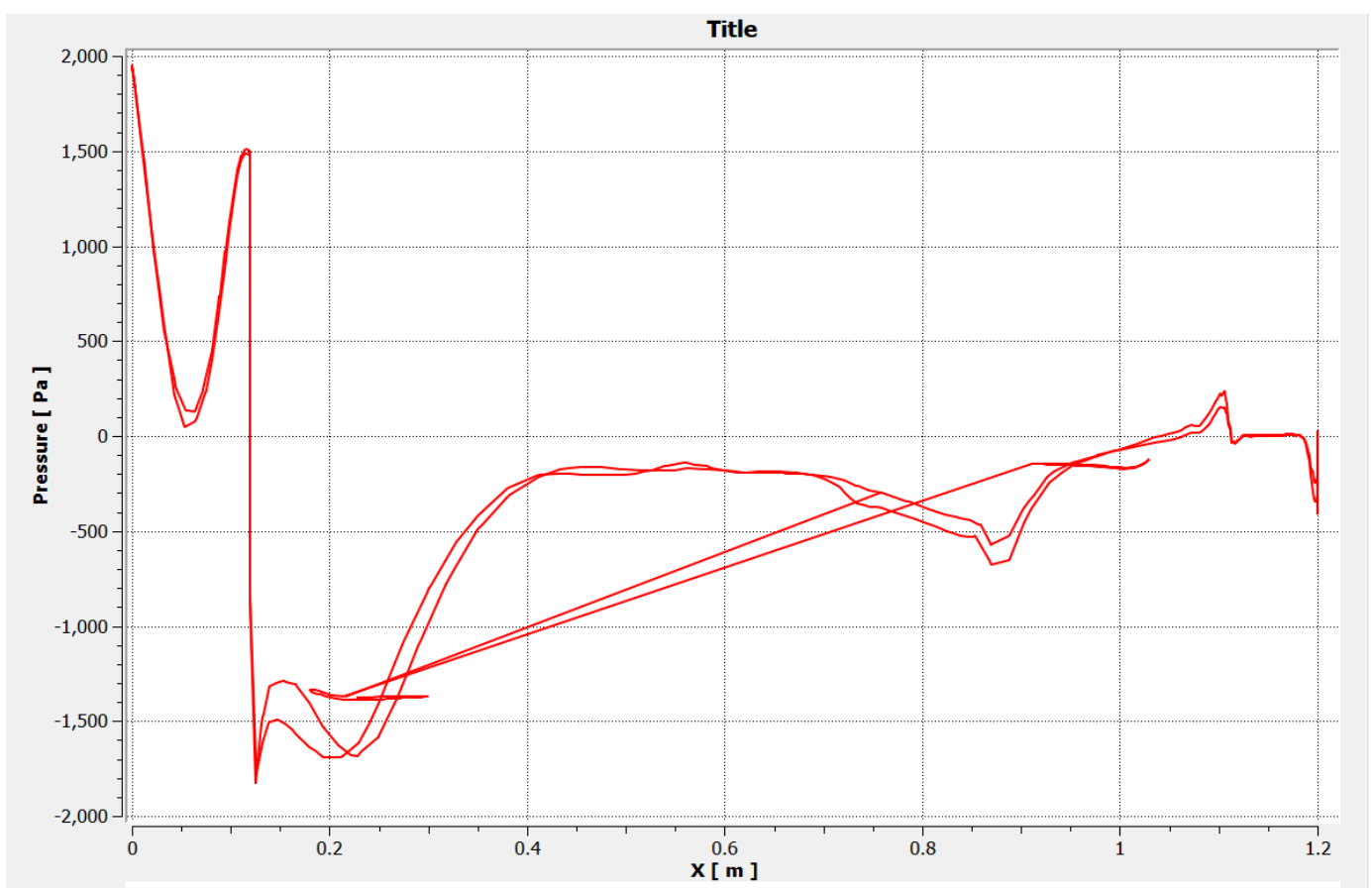

Figure. 12. Pressure distribution measured in equatorial plan of vehicle 


\section{Conclusions}

The study presented in this paper concentrated on a simulation about the distribution of pressure over vehicle body. The next step is to collect data about multiple sensors which could be attached to vehicle body.

The simulated result is going to be verified in the following with the experimental tests in basic and sea water. Future works could improve the accuracy of the CFD results by comparing the numerical simulation with the ROV using real-time adaptive identification approach in sea trial.

Vehicles can determine areas of interest for operation. The underwater vehicle have improve our ability to image the sea-floor, providing higher resolution seafloor mapping data. By transforming an underwater vehicle into a biomimetic vehicle to correct its own trajectory and stabilize its position, the exploitation and mapping capabilities of the marine environment increase both in quality and in reducing research time

\section{References}

[1]. Salumäe T, Kruusmaa M. Flow-relative control of an underwater robot. Proceedings of the Royal Society, 2013, 469, 20120671.

[2]. Nedelcu A, Clinci C. A survey of autonomous vehicles in scientific applications, "Mircea cel Batran" Naval Academy Scientific Bulletin, Volume XX - 2017 - Issue 2, p. 53-56.

[3]. Committee on Autonomous Vehicles in Support of Naval Operations Naval Studies Board Division on Engineering and Physical Sciences, Autonomous Vehicles in Support of Naval Operations, 2005;

[4]. Viedler, J. J., \& Hess, F. (1984). Fast continuous swimming of two pelagic predators, Saithe and Mackel: a kinematic analysis. Journal of Experimental Biology, 109, 209-228.

[5]. Eugen Rusu, Wave energy assessments in the Black Sea, Journal of Marine Science and Technology, September 2009, Volume 14, Issue 3, pp 359-372

[6]. Jin-Dong Liu și Huisheng Hu" Biologically Inspired Behaviour Design for Autonomous Robotic Fish" International Journal of Automation and Computing 4 (2006) 336-347

[7]. Jenhwa Guo, Maneuvering and control of a biomimetic autonomous underwater vehicle, Auton Robot (2009) 26: 241-249 DOI 10.1007/s10514-009-9117

[8]. O Tărăbuță, A Nedelcu, C Clinci and G Ichimoaei, A Study of the Feasibility of Autonomous Underwater Vehicles and Remotely Operated Vehicles in Black Sea, Scientific Bulletin of Naval Academy, Vol. XXI 2018, pg. 440-447.

[9]. M. W. J. Yuh, Underwater robotics, Third Edition,2001.

[10]. Dumitru Dinu, Constantin Vlad, Scafandri și Vehicule Subacvatice, Editura Științifică și Enciclopedică, București, 1986.

[11]. B.R. Clayton, R.E.D. Bishop, Mechanics of Marine Vehicles.

[12]. SolidWorks software

[13]. AnsysCFX software 